\title{
Transcatheter aortic valve implantation economics: a grisly reality
}

\author{
Antonis S. Manolis \\ Third Department of Cardiology, Athens University School of Medicine, Athens, Greece \\ Correspondence to: Antonis S. Manolis, MD. Vas. Sofias 114, Athens 115 27, Greece. Email: asm@otenet.gr.
}

\begin{abstract}
Transcatheter aortic valve implantation (TAVI), an established treatment for inoperable and highrisk operable symptomatic patients with severe aortic stenosis with growing numbers of procedures and expanding indications, is an expensive therapy. Cost-effectiveness analyses rely on the value of the incremental cost-effectiveness ratio (ICER), which is the difference in cost between two possible interventions, divided by the difference in their effect. Several analyses have demonstrated that TAVI is costeffective with an acceptable ICER for the inoperable patient alone and only via the iliofemoral route, while TAVI is more costly and is either less or equally effective as surgery in high-risk operable patients. When use of TAVI is extended to include a larger number of patients suitable for surgery, the overall results become less favorable. Acceptable ICERs should practically equate to the value of the gross domestic product (GDP) per capita in each country; however, the cost of the TAVI kit alone already exceeds the GDP per capita of all moderate- and low-income countries. An overview of the current cost-efficacy issues of TAVI is presented and this grisly reality is discussed, which may hopefully be improved in the future.
\end{abstract}

Keywords: Aortic stenosis; transcatheter aortic valve implantation (TAVI)/replacement; aortic valve replacement (AVR); cost-effectiveness; incremental cost-effectiveness ratio (ICER); gross domestic product (GDP); TAVI economics; quality of life (QoL)

Submitted Jun 05, 2017. Accepted for publication Jun 13, 2017.

doi: 10.21037/acs.2017.07.02

View this article at: http://dx.doi.org/10.21037/acs.2017.07.02

\section{Introduction}

Transcatheter aortic valve implantation (TAVI) has become an established treatment for inoperable ( $>50 \%$ probability of death) and high-risk (Society of Thoracic Surgeons-STS score $>8 \%$ ) symptomatic patients with severe aortic stenosis $(1-4)$. As the number of procedures is rapidly increasing worldwide $(5,6)$, the indications are also expanding to include intermediate-risk (STS score 4-8\%) patients $(3,7,8)$. Additionally, studies are now being designed to investigate its indication in even lower risk (STS score $<4 \%$ ) patients $(9,10)$. However, it is also universally recognized that TAVI is an expensive therapy, with a significantly higher upfront cost compared to surgical aortic valve replacement (AVR). Even the Americans have admitted that "TAVI is an expensive technology, and treating all eligible North American patients with TAVI would cost $>\$ 7$ billion" (11).

With any new treatment, the medical community should ask first if it is safe and clinically effective, which appears to be the case for TAVI, but also whether it is cost-effective, which still needs to be proven (12). From the outset, it can be said that TAVI may appear to be costeffective for the inoperable patient, but this may not hold true for all the other patients (13). Furthermore, even if it appears to be affordable for many countries in the developed world, its cost is prohibitive for the majority of the other nations. We will discuss TAVI economics in this brief overview and render our perspective on this costly therapeutic modality.

\section{Cost of TAVI}

Procedural costs are higher with TAVI, mainly due to the higher costs of the implant procedure $(11,14-17)$. However, nonprocedural costs are lower with TAVI compared with AVR, due to the shorter hospitalization period. The TAVI implant kit alone (valve, balloon, sheath) has a cost of $\$ 32,500$, while the surgical valve 
cost is only $\sim \$ 5,000$; similarly, in Switzerland the implant costs for the TAVI kit are $\sim \mathrm{CHF32,000} \mathrm{(} \mathrm{\$ 35,000),} \mathrm{while}$ the price for the surgical bioprosthesis is $\sim \mathrm{CHF} 3,000$ $(\$ 3,300)$ (2014 values) (18). Reimbursement in the USA is about \$40,000-\$45,000 depending on comorbidities and complications, and in Switzerland around CHF72,000 (\$78,000) for TAVI and CHF43,000 $(\$ 47,000)$ for AVR, which translates into a financial deficit for the hospitals performing TAVI.

On the basis of randomized data, TAVI in high surgicalrisk patients with the balloon-expandable valve was found to be cost-effective only via the iliofemoral, but not the transapical route. In the overall population, the incremental cost-effectiveness ratio (ICER) was $\$ 76,877$ per qualityadjusted life year (QALY) gained (17).

\section{Incremental cost-effectiveness ratio}

ICER is the difference in cost between two possible interventions, divided by the difference in their effect (19). It represents the average incremental cost associated with one additional unit of the measure of effect. The ICER can be estimated as:

ICER $=\left(\mathrm{C}_{1}-\mathrm{C}_{0}\right) /\left(\mathrm{E}_{1}-\mathrm{E}_{0}\right)$, where $\mathrm{C}_{1}$ and $\mathrm{E}_{1}$ are the cost and effect in the intervention group and where $\mathrm{C}_{0}$ and $\mathrm{E}_{0}$ are the cost and effect in the control care group, e.g., TAVI $v s$. standard therapy for inoperable patients with aortic stenosis, or TAVI vs. surgical AVR for high-risk aortic stenosis patients. A common application of the ICER is in cost-utility analysis, in which case the ICER is synonymous with the cost per QALY gained. The ICER can be used as a decision rule in resource allocation and varies greatly among countries (20), e.g., in the USA, an acceptable ICER is around $\$ 50,000$ (with a range up to $\$ 75,000$ ), while in the UK, the National Institute for Health and Care Excellence (NICE) adopts a nominal cost-per-QALY threshold of $£ 20,000$ to $£ 30,000$ (\$26,000-\$39,000), and in Canada an acceptable ICER hovers around C\$ 50,000 $(\sim 37,000)$.

\section{Cost-effectiveness}

As already mentioned, a cost-effectiveness analysis of the PARTNER study indicated that transfemoral (not transapical) TAVI in high surgical-risk patients with the balloon-expandable valve was cost-effective by the US standards with an ICER of \$76,877 per QALY gained (17). According with a similar study in inoperable patients, admission and short-term costs for TAVI were exuberant ( $\$ 78,000)$, while long-term 1-year follow-up costs were much lower compared with the medical therapy group, mainly due to lower re-hospitalization rates $(\sim \$ 18,000$ vs. \$44,000) (16). In the inoperable patient group, life expectancy with medical therapy was calculated at 1.23 years compared with 3.11 years offered by TAVI, yielding a difference of 1.88 years and a cost difference of $\$ 79,837$. This translates into an ICER of \$50,212 per life year gained. Other US studies have reported even higher ICERs reaching $\$ 116,500$ per QALY gained $(\$ 99,900$ per life-year gained) in inoperable patients (21).

Thus, TAVI is indeed very expensive to keep patients alive, which is the apparent reason why TAVI does not save money. The data on the route of access (transfemoral $v s$. transapical) have been consistent in indicating that the transapical TAVI has failed to meet criteria for acceptable ICER relative to $\operatorname{AVR}(17,22)$.

In a recent analysis of total costs of TAVI $(n=406)$ and $\operatorname{AVR}(n=710)$ in 1,116 patients, it was pointed out that the average direct costs to the hospital were $\$ 50,662$ for TAVI and $\$ 34,240$ for AVR, largely due to higher device costs (TAVI \$35,132 vs. AVR \$6,836) (23). Meanwhile, reimbursement was similar $(\$ 43,224$ for TAVI vs. $\$ 43,174$ for AVR).

According to a health technology assessment from the British National Institute for Health Research [2013], when first examining the patients not suitable for surgical AVRthe comparison in this case being simply between TAVI and medical management-the base-case results show TAVI as more costly yet more effective than the comparator, with an ICER below $£ 20,000$ (\$31,000) (24). For patients suitable for AVR, the comparator with TAVI is AVR and/ or medical management. In this case, TAVI is both more costly and less effective than the comparator in the basecase analysis, which assumes that the majority of patients in this group would receive AVR in the absence of TAVI. For example, when patients deemed suitable for AVR and $90 \%$ of them receive surgery, the ICER per QALY for this therapy is only $£ 13,000(\sim 20,000)$, while for patients suitable for TAVI and unsuitable for AVR (90\% receive TAVI) the ICER reaches $£ 31,000(\$ 48,000)$. However, when patients suitable for AVR (a less costly and more or equally effective therapy) actually receive TAVI, then the ICER is prohibitively high (24). Thus, when use of TAVI is extended to include a larger number of patients suitable for AVR, the overall results become much less favorable. More recent estimates from a real-world retrospective 


Table 1 Patient groups and measures that may keep TAVI cost-effective
Inoperable symptomatic patients with severe aortic stenosis with a predicted post-TAVI survival >1 year
High-risk operable patients (STS score >8)
Transfemoral approach
Strive for low-mortality and morbidity procedure (incl. minimizing paravalvular leak, stroke risk and need for ppm)
Minimalistic approach (see text for discussion)
Negotiate for lower price of the valve kit
ICER $\leq$ GDP per capita
Careful patient selection (e.g., shared decision making/life expectancy/end of life wishes)
GDP, gross domestic product; ICER, incremental cost-effectiveness ratio; ppm, permanent pacemaker; STS, Society of Thoracic
Surgeons; TAVI, transcatheter aortic valve implantation.

cohort analysis from the UK, indicated that the ICER for TAVI was $£ 10,533(\sim 15,500)$ per QALY, making it a costeffective procedure for inoperable patients with critical aortic stenosis (15). In another real-world data analysis, the ICER for the inoperable patients was $£ 17,718(\$ 26,000)$ per QALY gained and remained $<£ 30,000(\$ 44,000)$ per QALY gained in all sensitivity analyses performed (25). Some investigators have presented data showing that TAVI may be cost-effective even for high-risk operable patients as long as the ICER remains below the willingness-to-pay threshold set by NICE to <£20,000 (\$31,000) (12). Sensitivity analysis using the NICE threshold of $£ 20,000(\$ 31,000)$ showed TAVI to have a $64.6 \%$ likelihood of being cost-effective, compared with $35.4 \%$ for surgical AVR.

A US economic analysis used data from the US CoreValve High Risk Study (self-expandable valve), which randomized 795 high surgical risk patients (mean age 83 years, $53 \%$ male) with severe symptomatic aortic stenosis to TAVI or AVR (14). TAVI patients had lower 2-year mortality. Although both TAVI and AVR improved diseasespecific and health status at 1 month, only patients receiving iliofemoral TAVI had a significant health status benefit over AVR. This relative improvement was not sustained at 6 or 12 months. A relative benefit isolated to iliofemoral patients is consistent with findings from high-risk patients treated with the balloon-expandable valve. This study showed that lifetime ICERs were $\$ 55,090$ per QALY gained and $\$ 43,114$ per life year gained. The investigators found that the higher cost of the TAVI system (\$32,000 commercially) accounted for the largest proportion of TAVI expenses. Shorter ICU and hospital lengths of stay offset much of that charge, so that overall in-hospital TAVI costs were
$\$ 11,260$ more per patient over AVR. At 12 months, TAVI cost $\$ 9,207$ more than AVR. Sensitivity analysis examining the impact of reduced index TAVI admission costs on the ICER showed that reductions of $\sim \$ 1,650$ per admission would result in the ICER (TAVI $v s$. AVR) falling below the threshold of \$50,000 per QALY gained, which is considered to represent high economic value by the American standards.

Nation-specific economic assessments vary considerably. Neyt et al. (13) and Osnabrugge et al. (26) both reported exuberant ICER values for high-risk operable patients of $\$ 975,000(€ 750,000)$ and \$232,128 (€150,000) in Belgium and the Netherlands respectively, compared with $\$ 76,877$ reported by Reynolds et al. (17) in the USA. For inoperable patients, the ICER was on average $\$ 58,000$ (€45,000) per QALY and \$62,000 (€48,000) per year gained, respectively. In the British study mentioned above, the ICER was $\$ 21,400$ $(£ 12,900)$ per QALY for the inoperable patients and $\$ 112,000(£ 68,000)$ for high-risk operable patients (24).

Thus, TAVI may be economically justifiable when compared with medical therapy for ineligible surgical candidates according to reported ICERs (Table 1) $(13,27)$. However, for high-risk patients who were eligible for surgery, the evidence was not as strong, and significant variations were identified depending on the healthcare system setting, procedural approach, and patient selection process (13). Perhaps one of the most important determinants of the overall cost, other than the transcatheter valve device itself, was the incidence of periprocedural complications (28). Approximately $15 \%$ of TAVI procedures appear to be performed in nonagenarians, in whom the procedure is feasible, albeit at 
increased mortality and stroke risk; however, arguments are emerging as there is concern that the ICER for TAVI in nonagenarians may exceed the societal willingness-to-pay threshold and reach prohibitive levels such as $\$ 100,000$ or even \$150,000 per QALY gained (29).

\section{Periprocedural morbidity and mortality}

Indeed, several studies reported sensitivity analyses that predicted significant improvements in the costeffectiveness of TAVI, if it could be performed with lower periprocedural morbidity and mortality (28). Arnold et al. presented multivariable models using detailed data from 406 of 519 (78\%) participants from the PARTNER I trial to estimate the independent impact of periprocedural complications on in-hospital costs and duration of hospitalization for patients who underwent TAVI (28). Almost half (49\%) of all patients had $\geq 1$ complication during their index hospitalization. Patients who had a complication were more likely to be women and belonging to cohort B (inoperable patients), but less likely to have had previous "bypass" surgery. For these patients, the unadjusted incremental cost was $\$ 33,196$, with an incremental duration of hospitalization of 6.6 days, significantly higher than those patients who did not have a complication. Specifically, seven complications were found to be associated with significant increases in hospitalization costs; a repeat TAVI procedure, death, renal failure, major bleeding, surgical conversion, major stroke, and major arrhythmias leading to permanent pacemaker implantation. Death, renal failure, major bleeding, vascular complications, major arrhythmia, and pacemaker implantation were the complications most predictive of a prolonged length of stay, and an estimated 2.4 days of the overall duration of hospitalization were directly attributable to periprocedural complications. Thus, in the PARTNER trial, periprocedural complications were frequent and costly accounting for $\$ 12,475$ per patient in initial hospital costs and 2.4 days of hospitalization. This translated into $1 / 4$ of non-implant-related hospital costs (28). A recent observational study from Germany indicated that post procedural complications after TAVI, such as bleeding events, acute kidney injuries, and strokes were associated with increased resource use and need for substantial amounts of additional reimbursement (30).

Compared with surgery, TAVI has been plagued by higher rates of paravalvular leaks and pacemaker implantations (31), both of which add to increased local resource usage and costs. Thus, TAVI technology and implantation techniques need to address these issues in order to lower the cost compared to AVR.

\section{Minimalistic approach}

An initial standard approach to TAVI included transfemoral, transapical or other vascular site access, use of general anesthesia and use of transesophageal echocardiogram for guidance, performed either in the cardiac catheterization laboratory or in a hybrid operating room. For vascular site access, either a percutaneous approach with use of vascular closure device for hemostasis or cut-down surgical technique was employed. In some laboratories, even a pulmonary artery catheter was used for monitoring. However, this has subsequently developed into a minimalist approach with mainly percutaneous transfemoral access site entry, use of local anesthesia and/or conscious sedation (with use of fentanyl, midazolam and/or propofol), without echo guidance and with use of percutaneous pre-closure hemostatic device(s) for vessel closure (32).

Comparative studies of the standard and minimalist approaches indicate that in the current era, given the advent of smaller-sized sheaths and valve profiles, the minimalist transfemoral approach can be performed with minimal morbidity and mortality and equivalent effectiveness compared with the standard approach $(33,34)$. Furthermore, a shorter length of hospital stay and lower resource use with the latter approach significantly lowers hospital costs.

\section{Incremental cost-effectiveness ratio and gross domestic product per capita}

According to the WHO-CHOICE (World Health Organization-CHOosing Interventions that are CostEffective) project, a WHO initiative developed in 1998 with the objective of providing policy makers with evidence for deciding on interventions and programs which maximize health for the available resources, interventions are highly cost-effective when they have an ICER below the gross domestic product (GDP) per capita, cost-effective if the ICER is $1-3$ times the GDP per capita, and not costeffective when the ICER is $>3$ times the GDP per capita (35). However, even the most affluent countries consider that an acceptable ICER should be around the GDP per capita and generally $<1.5$ times the GDP per capita.

With these caveats in mind, each country has to calculate 


\begin{tabular}{|c|c|c|c|}
\hline Australia & $\$ 56,000$ & $\$ 56,000$ & $\$ 56,000-\$ 84,000$ \\
\hline Brazil & $\$ 8,680$ & $\$ 8,680$ & $\$ 8,680-\$ 13,000$ \\
\hline Canada & $\$ 43,300$ & $\$ 43,300$ & $\$ 43,300-\$ 65,000$ \\
\hline Czech Republic & $\$ 17,500$ & $\$ 17,500$ & $\$ 17,500-\$ 26,000$ \\
\hline France & $\$ 36,300$ & $\$ 36,300$ & $\$ 36,300-\$ 54,500$ \\
\hline Germany & $\$ 41,200$ & $\$ 41,200$ & $\$ 41,200-\$ 62,000$ \\
\hline Greece & $\$ 18,000$ & $\$ 18,000$ & $\$ 18,000-\$ 27,000$ \\
\hline Poland & $\$ 12,500$ & $\$ 12,500$ & $\$ 12,500-\$ 19,000$ \\
\hline Russia & $\$ 9,300$ & $\$ 9,300$ & $\$ 9,300-\$ 14,000$ \\
\hline Switzerland & $\$ 81,000$ & $\$ 81,000$ & $\$ 81,000-\$ 121,000$ \\
\hline UK & $\$ 44,000$ & $\$ 44,000$ & $\$ 44,000-\$ 66,000$ \\
\hline USA & $\$ 56,000$ & $\$ 56,000$ & $\$ 56,000-\$ 84,000$ \\
\hline High-income countries & $\$ 40,000$ & $\$ 40,000$ & $\$ 40,000-\$ 60,000$ \\
\hline Middle-income countries & $\$ 5,000$ & $\$ 5,000$ & $\$ 5,000-\$ 7,500$ \\
\hline Low-income countries & $\$ 600$ & $\$ 600$ & $\$ 600-\$ 900$ \\
\hline
\end{tabular}

its own ICER in close association with the GDP per capita. From the list of GDPs per capita for various countries (Table 2), one can easily identify the size of the problem imposed on societies by the current cost of TAVI, which is apparently prohibitive for many nations (middle- and low-income countries). Several countries have already established their willingness-to-pay thresholds for TAVI, e.g., the US has set a $\$ 50,000$ bar per QALY (19), the UK NICE has set a $£ 20,000(\$ 31,000)$ willingness-to-pay threshold per QALY gained (12), in Canada, such value is between 20,000 and 40,000 Canadian dollars $(\$ 14,400$ $\$ 29,000)$, and in the Netherlands at $<€ 80,000(\$ 90,000)$.

However, even in high-income countries, TAVI is not cost-effective at the societal cost-effectiveness threshold of $<\$ 50,000$ per QALY, when life expectancy after TAVI is $<3$ years, when quality of life $(\mathrm{QoL})$ is not improved after TAVI, or when surgical AVR is equally effective and less costly. Furthermore, within the same country (the US), there is great heterogeneity in GDP per capita that a uniform ICER that equals the country's GDP per capita exceeds the budget of many individual states (36). Thus, until the valve and its paraphernalia become cheaper, TAVI will still remain a non-affordable luxury for many patients. Nevertheless, in the long run it may be more ethical to consider the argument put forth by investigators about setting economical barriers to available therapies who state that "an ICER threshold is better a guide for understanding value rather than as a willingness-topay barrier", but who also agree that "cost-effectiveness (analysis) can help resolve whether expensive new therapy, while claiming to offer greater effectiveness, also offers good value" (37).

\section{Quality of life}

Typically, patients currently referred for and treated by TAVI are elderly with a concomitant multitude of comorbidities and disabilities (38). They have limited life 
expectancy, as they have most likely already surpassed the average life expectancy in their country. Beyond safety and efficacy, the assessment of health-related quality of life (QoL) is of great importance not only to guide shared clinical decision-making, but also to evaluate this new treatment modality in this high-risk patient population. In their QoL study from the German TAVI registry, Lange et al. included 2,288 patients (transvascular TAVI, $\mathrm{n}=1,626$; transapical TAVI, $\mathrm{n}=662$ ), who had in-hospital mortality of $5.9 \%$ and a 1 -year mortality of $23 \%$ (38). They reported, in parallel to an improvement in NYHA functional class, QoL improvement in about two thirds of patients. However, using other validated QoL instruments assessing activity, self-care, pain or discomfort and anxiety or depression at 1 year, they reported improvement by at least one level in only $20 \%$, unchanged QoL status in 40-60\%, while QoL worsened for $\sim 20 \%$ of patients. The authors concluded that there was a sizeable group of patients who did not derive any QoL benefits. Several independent pre- and postoperative factors were identified as being predictive for less pronounced QoL benefits.

In another recent QoL study comprising 7,014 surviving TAVI patients $(51 \%$ men; median age 84 years, range $78-88), 62.3 \%$ had a favorable outcome at 1 year (39). The lowest QoL rates were seen among patients with severe chronic obstructive pulmonary disease (COPD) $(51.4 \%)$, those on dialysis $(47.7 \%)$, or those with very poor baseline health status (49.2\%). The authors concluded that although the health status results were favorable for most patients, approximately 1 in 3 still have a poor outcome 1 year after TAVI.

\section{Conclusions}

A recent systematic review of 14 decision-analytic models regarding the cost-effectiveness analyses of heart valve implantations describes the gist of this presentation: in most studies, TAVI was cost-effective compared to standard treatment in inoperable or high-risk operable patients, albeit with a high variation of the ICER range $(€ 18,421-$ $€ 120,779)(\$ 21,000-\$ 136,480)(40)$. In all studies, surgical AVR was cost-effective compared to standard therapy in operable patients [ICER range €14,108-€40,944 (\$16,000$\$ 46,000)]$, but the results were not consistent on the costeffectiveness of TAVI $v s$. AVR in high-risk operable patients (ICER range: dominant to dominated by AVR). These results are in keeping with all prior studies indicating that AVR is much more cost-effective than TAVI in high- risk patients. Thus, the question remains whether and when TAVI will become cost-effective in these high-risk operable patients in comparison with AVR, and more importantly whether TAVI will ever be cost-effective for intermediate and low-risk patients, even if it is demonstrated to be equally safe and effective with AVR. Importantly, most data presented herein come from the US and Western Europe, and obviously, findings based on these healthcare systems may be less applicable to other countries. Thus, nationspecific economic assessments of the cost-effectiveness of TAVI could be performed in the future that may guide and optimize source utility in each country. Finally, QoL does not seem to improve in all TAVI patients, as 1 in 3 patients still have a poor outcome at 1 year, which raises questions as to the long-term efficacy of TAVI and/or the process of patient selection.

\section{Perspective}

TAVI has been demonstrated to be the treatment of choice for inoperable patients when they have a predicted postTAVI survival of $>1$ year. It is in this patient group that TAVI has been shown consistently to be cost-effective, with an acceptable ICER per QALY. Based on the comparative results between TAVI and AVR, TAVI appears equally effective and safe with AVR in both high- and intermediatesurgical risk symptomatic patients with severe aortic stenosis. However, from the preceding discussion it has become abundantly clear that for high-risk operable patients, TAVI is currently a more expensive therapy and probably a less effective alternative to surgical AVR, with an ICER that may be acceptable for high-income countries, but definitely not for the moderate- or low-income countries. When use of TAVI is extended to include a larger number of moderate- to low-risk patients suitable for AVR, overall results become less favorable. Only reduced index TAVI procedure costs will render this non-surgical modality appealing to and applicable in larger groups of patients and in more countries. When manufacturers reduce the exuberant cost of the valve and its accessories, TAVI may become the predominant therapy for patients with severe aortic stenosis. This course of action may be further realized if this technology can be employed with a minimalistic approach at reduced complication rates, when improved QoL is demonstrated for these patients, and when the valves prove durable in the long-term. All these conditions do appear to be within the realm of feasibility, hopefully in the near future. 


\section{Acknowledgements}

None.

\section{Footnote}

Conflicts of Interest: The author has no conflicts of interest to declare.

\section{References}

1. Vahanian A, Alfieri O, Andreotti F, et al. Guidelines on the management of valvular heart disease (version 2012). Eur Heart J 2012;33:2451-96.

2. Otto CM, Kumbhani DJ, Alexander KP, et al. 2017 ACC Expert Consensus Decision Pathway for Transcatheter Aortic Valve Replacement in the Management of Adults With Aortic Stenosis: A Report of the American College of Cardiology Task Force on Clinical Expert Consensus Documents. J Am Coll Cardiol 2017;69:1313-46.

3. Nishimura RA, Otto CM, Bonow RO, et al. 2017 AHA/ ACC Focused Update of the 2014 AHA/ACC Guideline for the Management of Patients With Valvular Heart Disease: A Report of the American College of Cardiology/ American Heart Association Task Force on Clinical Practice Guidelines. Circulation 2017;135:e1159-95.

4. Manolis AS, Polydorou A, Rouska E, et al. Transcatheter aortic valve iplantation in severe left ventricular dysfunction: A viable option in a patient with low-flow, low-gradient critical aortic stenosis. Hosp Chronicles 2013;8:97-9.

5. Vahl TP, Kodali SK, Leon MB. Transcatheter Aortic Valve Replacement 2016: A Modern-Day "Through the Looking-Glass" Adventure. J Am Coll Cardiol 2016;67:1472-87.

6. Eggebrecht H, Mehta RH. Transcatheter aortic valve implantation (TAVI) in Germany 2008-2014: on its way to standard therapy for aortic valve stenosis in the elderly? EuroIntervention 2016;11:1029-33.

7. Leon MB, Smith CR, Mack MJ, et al. Transcatheter or Surgical Aortic-Valve Replacement in Intermediate-Risk Patients. N Engl J Med 2016;374:1609-20.

8. Reardon MJ, Van Mieghem NM, Popma JJ, et al. Surgical or Transcatheter Aortic-Valve Replacement in Intermediate-Risk Patients. N Engl J Med 2017;376:1321-31.

9. The PARTNER 3 - Trial - The Safety and Effectiveness of the SAPIEN 3 Transcatheter Heart Valve in Low
Risk Patients With Aortic Stenosis (P3). Available online: https://clinicaltrialsgov/ct2/show/NCT02675114

10. Medtronic Transcatheter Aortic Valve Replacement in Low Risk Patients. Available online: https://clinicaltrials. gov/ct2/show/NCT02701283

11. Dhruva SS, Krumholz HM. The Core Value of CostEffectiveness Analyses. J Am Coll Cardiol 2016;67:39-41.

12. Fairbairn TA, Meads DM, Hulme C, et al. The costeffectiveness of transcatheter aortic valve implantation versus surgical aortic valve replacement in patients with severe aortic stenosis at high operative risk. Heart 2013;99:914-20.

13. Neyt M, Van Brabandt H, Devriese S, et al. A costutility analysis of transcatheter aortic valve implantation in Belgium: focusing on a well-defined and identifiable population. BMJ Open 2012;2:e001032.

14. Reynolds MR, Lei $Y$, Wang K, et al. Cost-Effectiveness of Transcatheter Aortic Valve Replacement With a SelfExpanding Prosthesis Versus Surgical Aortic Valve Replacement. J Am Coll Cardiol 2016;67:29-38.

15. Freeman PM, Protty MB, Aldalati O, et al. Severe symptomatic aortic stenosis: medical therapy and transcatheter aortic valve implantation (TAVI)-a realworld retrospective cohort analysis of outcomes and cost-effectiveness using national data. Open Heart 2016;3:e00414.

16. Reynolds MR, Magnuson EA, Wang K, et al. Costeffectiveness of transcatheter aortic valve replacement compared with standard care among inoperable patients with severe aortic stenosis: results from the placement of aortic transcatheter valves (PARTNER) trial (Cohort B). Circulation 2012;125:1102-9.

17. Reynolds MR, Magnuson EA, Lei Y, et al. Costeffectiveness of transcatheter aortic valve replacement compared with surgical aortic valve replacement in highrisk patients with severe aortic stenosis: results of the PARTNER (Placement of Aortic Transcatheter Valves) trial (Cohort A). J Am Coll Cardiol 2012;60:2683-92.

18. Jeger $R, T$ Toggweiler $S$. Interventional treatment for structural heart disease: who is deciding, and can we afford it? Swiss Med Wkly 2014;144:w14046.

19. Anderson JL, Heidenreich PA, Barnett PG, et al. ACC/ AHA statement on cost/value methodology in clinical practice guidelines and performance measures: a report of the American College of Cardiology/American Heart Association Task Force on Performance Measures and Task Force on Practice Guidelines. J Am Coll Cardiol 2014;63:2304-22. 
20. Nanavaty M, Kaura S, Mwamburi M, et al. The use of incremental cost-effectiveness ratio thresholds in health technology assessment decisions. J Clin Pathways 2015;1:29-36.

21. Simons CT, Cipriano LE, Shah RU, et al. Transcatheter aortic valve replacement in nonsurgical candidates with severe, symptomatic aortic stenosis: a cost-effectiveness analysis. Circ Cardiovasc Qual Outcomes 2013;6:419-28.

22. Gada H, Agarwal S, Marwick TH. Perspective on the costeffectiveness of transapical aortic valve implantation in high-risk patients: Outcomes of a decision-analytic model. Ann Cardiothorac Surg 2012;1:145-55.

23. Arsalan M, Ballard A, MacLachlan CR, et al. Comparison of Current Costs and Reimbursement for Transcatheter and Surgical Aortic Valve Replacement. J Am Coll Cardiol 2016;67:10.

24. Orlando R, Pennant M, Rooney S, et al. Cost-effectiveness of transcatheter aortic valve implantation (TAVI) for aortic stenosis in patients who are high risk or contraindicated for surgery: a model-based economic evaluation. Health Technol Assess 2013;17:1-86.

25. Brecker S, Mealing S, Padhiar A, et al. Cost-utility of transcatheter aortic valve implantation for inoperable patients with severe aortic stenosis treated by medical management: a UK cost-utility analysis based on patientlevel data from the ADVANCE study. Open Heart 2014;1:e000155.

26. Osnabrugge RL, Head SJ, Genders TS, et al. Costs of transcatheter versus surgical aortic valve replacement in intermediate-risk patients. Ann Thorac Surg 2012;94:1954-60.

27. Indraratna $P, A n g$ SC, Gada H, et al. Systematic review of the cost-effectiveness of transcatheter aortic valve implantation. J Thorac Cardiovasc Surg 2014;148:509-14.

28. Arnold SV, Lei Y, Reynolds MR, et al. Costs of periprocedural complications in patients treated with transcatheter aortic valve replacement: results from the Placement of Aortic Transcatheter Valve trial. Circ Cardiovasc Interv 2014;7:829-36.

29. Weintraub WS. TAVR in Nonagenarians: Pushing the Boundaries. J Am Coll Cardiol 2016;67:1396-8.

30. Kaier K, Reinecke H, Naci H, et al. The impact of post- procedural complications on reimbursement, length of stay and mechanical ventilation among patients undergoing transcatheter aortic valve implantation in Germany. Eur J Health Econ 2017. [Epub ahead of print].

31. Manolis AS. Need for a permanent pacemaker after transcatheter aortic valve implantation (TAVI). Rhythmos 2016;11:87-9.

32. Colombo A, Michev I, Latib A. Is it time to simplify the TAVI procedure? "Make it simple but not too simple". EuroIntervention 2014;10 Suppl U:U22-7.

33. Babaliaros V, Devireddy C, Lerakis S, et al. Comparison of transfemoral transcatheter aortic valve replacement performed in the catheterization laboratory (minimalist approach) versus hybrid operating room (standard approach): outcomes and cost analysis. JACC Cardiovasc Interv 2014;7:898-904.

34. Toppen W, Johansen D, Sareh S, et al. Improved costs and outcomes with conscious sedation vs general anesthesia in TAVR patients: Time to wake up? PLoS One 2017;12:e0173777.

35. Cost effectiveness and strategic planning (WHOCHOICE). Available online: http://www.who.int/choice/ cost-effectiveness/en/

36. Diamond GA, Kaul S. Cost, effectiveness, and costeffectiveness. Circ Cardiovasc Qual Outcomes 2009;2:49-54.

37. Weintraub WS, Cohen DJ. The limits of cost-effectiveness analysis. Circ Cardiovasc Qual Outcomes 2009;2:55-8.

38. Lange R, Beckmann A, Neumann T, et al. Quality of Life After Transcatheter Aortic Valve Replacement: Prospective Data From GARY (German Aortic Valve Registry). JACC Cardiovasc Interv 2016;9:2541-54.

39. Arnold SV, Spertus JA, Vemulapalli S, et al. Qualityof-Life Outcomes After Transcatheter Aortic Valve Replacement in an Unselected Population: A Report From the STS/ACC Transcatheter Valve Therapy Registry. JAMA Cardiol 2017;2:409-16.

40. Huygens SA, Takkenberg JJ, Rutten-van Molken MP. Systematic review of model-based economic evaluations of heart valve implantations. Eur J Health Econ 2017. [Epub ahead of print].
Cite this article as: Manolis AS. Transcatheter aortic valve implantation economics: a grisly reality. Ann Cardiothorac Surg 2017;6(5):516-523. doi: 10.21037/acs.2017.07.02 